\title{
Application of multimedia technology in the doctor-patient communication in kidney transplantation department
}

\author{
Jinguo Wang ${ }^{1,}$, the corresponding author: Na Wang ${ }^{2, b}$ \\ and Yang $\mathrm{Gao}^{3, \mathrm{C}}$ \\ ${ }^{1}$ Department of Urology, the First Hospital of Jilin University, China \\ ${ }^{2}$ Department of Anesthesiology, the First Hospital of Jilin University, China \\ ${ }^{3}$ Department of Anesthesiology, the First Hospital of Jilin University, China \\ awangjinguolily@163.com, ㅎangna080613@163.com, 975707998@qq.com ${ }^{c}$
}

\begin{abstract}
Keywords: urology, multimedia, doctor-patient communication.
Abstract. According to the characteristics of diseases of urology, doctor-patient communication can be applied individually in accordance with patients' comprehension. Improve the understanding of diseases. The development of multimedia technology makes the isolation ward is no longer isolated space, use of multimedia can connect patients with their families and the outside world, reduce the occurrence of the care unit syndrome and promote the patient's satisfaction. Kidney transplant is a permanent chronic disease, patients need lifelong treatment in the complicated society, this requires a kidney transplant patient often keep in touch with the physician and other patients, establish a new mode of doctor-patient interaction, and gradually implement self-management of chronic diseases.
\end{abstract}

\section{Introduction}

Kidney transplantation is a kind of surgery to transplanta healthy kidney to a patient with end-stage kidney disease and loss of renal function. Renal transplant which is a treatment of uremia besides dialysis has been more successful performed for many patients. Kidney transplant patients will take immunosuppressants, as long as the transplanted kidney has function.

\section{Organization of the Text}

After kidney transplant patients are generally treated in care unit for observation for 3 to 5 days, during which patients will be separated from his family, who will also contain drainage tube, central venous catheter, etc. At the same time because of discomfort and pain after surgery and the special environment. The patients may fell nervous and fear. In the elderly it is more apparent. In order to make effective communication between doctors and patients, our department use the multimedia technology which has obtained the good effect.

We communicate patients not only according to the past with language communication by doctors and nurses, but also on the basis of multimedia input sound and painting pictures, disease and health education slides, pain knowledge, immunosuppressive therapy and the requirements of the the therapy, pictures of patients' family which are up to patients to choose their favorite part of the content. When the patients leave the isolated wards, the questionnaire survey is used to evaluate patients' satisfaction and the degree of the cognition to the disease.

Questionnaire fully confirm that multimedia communication and effective. Multimedia technology is more vivid, more intuitive to replace the doctor- patient communication and education, to avoid communication problems resulting from the language barrier or other occurring in the course of communication. Patients at the same time actively participate in the communication. It also reduce the work burden of the doctor's and save time for them to treat illness, make communication more effective. The patient's satisfaction is higher. 
The development of information technology makes the isolation ward is no longer isolated space, use of multimedia can connect patients with their families and the outside world, reduce the occurrence of the care unit syndrome and promote the patient's satisfaction.

It is the key problem to improve the service quality because of tension in doctor-patient relationship of medical environment. We must master every link in our service which is very important to prevent medical disputes. Multimedia communication in isolation unit not only reflects "all for the aim of serving patients", but also keeps the corresponding information, increases the trust from the patients and their families. There have been reports that in the isolation close relatives should company the patients, give patients mental support, but the existing rules of the isolation ward still do not allow accompany. Besides in order to reduce the occurrence of nosocomial infection, other people who are not involved in the patients' treatment are not allowed to stay in the isolation wards. Multimedia technology can promote patients recover. Although in the initial stage constructing of multimedia technology is time and energy-consuming, but in the later using process it can not only improve the effect, but also save the physical strength and energy. The platform can be used repeatedly for many years, making the patient get the high quality service.

To effectively improve the quality of life in patients undergoing renal transplant and introduce self-management methods to renal transplant receptor through multimedia, our hospital carry out doctor-patient interactive health education, and good effects have been achieved. After regular follow-up for 1 year in this study, we adopt the doctor-patient interactive health education, specific measures as follows: regularly organizing patients to participate in the lecture in the internet once a month and $1 \mathrm{~h}$ every time; using multimedia, discussion and experience exchange, fellowship, video and game to systematically demonstrate pathogenesis and inducement, complications of the immunosuppression therapy and treatment method, and the matters needing attention of drug treatment. Through regular home visits or $24 \mathrm{~h}$ open calls, we get the information feedback which is targeted to the health education to patients, and then give specific reasonable instructions and effective consultation [1].

As medical and health system reform and the enhancement of people's health consciousness, the traditional medical service mode is difficult to meet people's growing health needs. The medical treatment and management in patients undergoing renal transplantation will be a lifetime-long process. The purpose of education for renal transplant patients is to make the knowledge of renal transplantation popular, inspire patients' initiative, actively cooperate with medical staff to control the disease. The traditional health education only focuses on the learning of theoretical knowledge, only to impart the extensive knowledge to patients, and do not pay attention to the feedback of information, so the patient is in a state of passive learning. This method of teaching can't stimulate the enthusiasm of patients with active participation[2].

Renal transplant patients can't take part in too much the public issues, because of immunotherapy, so they may be isolated from the society. Multimedia provided them with opportunities to contact with doctors and other patients to talk more about their illness and experience.

Among them, the interactive multimedia and interactive multimedia system design and optimization is very important to improve the user experience. On the one hand, interactive multimedia applications has been increasing popularity in People's Daily lives, such as games, teaching, multimedia conferences, etc. On the other hand, users want to obtain the multimedia service with more rich content, higher visual quality, better interactivity, and can be more convenient access. For multimedia system, richer content to have more storage, transmission and processing capacity; higher visual quality requirements more efficient multimedia compression, transmission and processing algorithms; better interactive end-to-end delay requirements as low as possible; and more convenient access to insist on multimedia system can adapt to the continuous development of heterogeneous client device and then into the user's daily life. Therefore, how to design according to the above requirements and optimization of interactive multimedia system become the difficulties and challenges we face[3]. 
This article set out from the current background and tendency of the development of multimedia technology and computing devices, studies the advanced architecture of the remote interactive multimedia system design and optimization of the modules. Through the use of remote computing, virtual display, collaborative technologies such as more equipment, greatly improve the user experience of remote interactive multimedia system, also extends the application field of interactive multimedia services. In this paper, the specific research content and the innovation place mainly includes:

The scheme based on color quantization and fixed length coding, the existing various source texture format for hardware friendly texture compression. It is suitable for high dynamic range texture, texture, transparency, low dynamic range, etc. In particular, according to the characteristics of different source texture format, this paper designed the data rate of fixed length coding compression algorithm, and support the rapid transformation between different compressed format, which can meet the requirements of rendering systems with different abilities [4]. For remote interactive multimedia system, uniform texture compression scheme not only can better support in thin client devices, especially mobile and handheld devices on the user interface rendering synthesis, and compression texture greatly saves the background of storage virtualization module and processing overhead, so as to improve the capacity and performance of the system, and can provide more users with more rich content of interactive multimedia services.

Put forward for the condensation of $2 \mathrm{~d}$ image elements and post-processing algorithm. In the remote interactive multimedia system proposed in this paper, all kinds of typical interactive multimedia application after dealing with the system background of virtualization module, are represented as one or more of the two-dimensional image sequence and interactive control command flow elements. Therefore, this article designed for the condensation of $2 \mathrm{~d}$ image elements and post-processing algorithm. First of all, this paper puts forward for mixed images, such as screen image real-time compression and scaling algorithm, which based on the real-time content of mixed image detection, using the image area with different content of different adaptive compression, compression characteristics and the content of the image area for the corresponding adaptive post-processing realize scale operation. At the same time, this article also use graphics processor to further accelerate hybrid image real-time zooming. Secondly, on the other kind of $2 \mathrm{~d}$ graphic elements, namely high dynamic range image, especially in high dynamic range of medical image, is proposed in this paper based on the values of interest embedded bit plane compression algorithm. Interested in using Columbus gray coding realized it support scalable values gradually transfer, at the same time can accurately estimate the truncation error in transmission.

Designed and built a heterogeneous client device oriented interactive virtual display system. Using the texture compression scheme and image elements in front of the compression and post-processing algorithm, this paper realized the remote computing based on thin client and more collaborative remote virtual display system equipment. The system architecture consists of portable mobile devices, wearable sensors, input and output devices in one's area and environment, and remote computing resources, etc. In this framework, the background server virtualization module, the middle layer of transmission and control module, as well as the client's synthesis and display module to achieve the efficient remote interactive virtual display. By make full use of the computing resources of remote and heterogeneous client device, this paper puts forward an interactive virtual display system has good mobility, interactivity, and computing and storage performance, can make use of the existing thin client computing devices support interactive multimedia applications with rich user experience. Established a new remote interactive multimedia services, i.e., according to the user as the center of remote digital visual health service. This article will further specialization of remote interactive multimedia system is proposed to support different digital health applications, using sensors, client devices, server work together. In a typical digital health system architecture, we proposed to the digital 3 d virtual human body health data representation method, and applied to system on the backend server virtualization module. Through remote virtual display, a variety of health data can be unified virtual human visual services and the use of heterogeneous client device user interaction in 
real time. In this paper, based on the remote rendering and virtual display technology of virtual human body visualization scheme can be a variety of online digital medical services (such as query, health monitoring, and clinical information browsing, etc.), so as to promote the development of digital health systems and services.

\section{Conclusions}

Therefore, multimedia interactive education is the effective ways to improve the patients quality of life and confidence. Renal transplant patients learn knowledge of renal transplantation and prevent or delay the occurrence and development of complications, reduce the economic burden of family and society.

Anyhow, kidney transplant is a permanent chronic disease, patients need lifelong treatment in the complicated society, this requires a kidney transplant patient often keep in touch with the physician and other patients, establish a new mode of doctor-patient interaction, and gradually implement self-management of chronic diseases. The rapid development of information and communications technology for today's multimedia technology research has brought many new topics.

\section{References}

[1] Rajab LD, Baqain ZH. Use of information and communication technology among dental students at the University of Jordan. J Dent Educ. 69, 387-98 (2005).

[2] Link TM, Marz R. Computer literacy and attitudes towards e-learning among first year medical students. BMC Med Educ. 19, 34 (2006).

[3] Hendricson W, Eisenberg E, Guest G, Jones P, Johnson L, Panagakos F, McDonald J, Cintron L. What do dental students think about mandatory laptop programs? J Dent Educ. 70, 480-99 (2006).

[4] Seago BL, Schlesinger JB, Hampton CL. Using a decade of data on medical student computer literacy for strategic planning. J Med Libr Assoc. 90,202-9 (2002). 